\title{
Erratum: Einstein-Cartan Portal to Dark Matter [Phys. Rev. Lett. 126, 161301 (2021)]
}

Mikhail Shaposhnikov, Andrey Shkerin, Inar Timiryasov@, and Sebastian Zell

(Received 14 September 2021; published 15 October 2021)

DOI: 10.1103/PhysRevLett.127.169901

In [1], an additional higher order term was missing in the scalar-fermion interaction, and the overall sign of the fourfermion terms was flipped. As a result, the overall sign of Eq. (2) in [2] should be flipped. This does not affect any results in the main text of the Letter.

The following changes must be applied to Appendix A of [2] (presented in the Supplemental Material). (i) The overall sign of Eq. (A2) should be flipped. (ii) Equation (A3) must read

$$
\begin{aligned}
S_{s f}= & \int \mathrm{d}^{4} x \sqrt{-g} \frac{3 \alpha}{4}\left[\frac{\partial_{\mu} \Omega^{2}}{\Omega^{2}}+\frac{\gamma}{\gamma^{2}+1}\left(\frac{\partial_{\mu} \eta}{\Omega^{2}}+\partial_{\mu} \gamma\right)\right] V^{\mu} \\
& +\int \mathrm{d}^{4} x \sqrt{-g} \frac{3}{4}\left[\beta \frac{\partial_{\mu} \Omega^{2}}{\Omega^{2}}+\frac{1+\gamma \beta}{\gamma^{2}+1}\left(\frac{\partial_{\mu} \eta}{\Omega^{2}}+\partial_{\mu} \gamma\right)\right] A^{\mu},
\end{aligned}
$$

with $\Omega^{2}=1+\left(\xi h^{2} / M_{P}^{2}\right)$. (iii) Equation (A6) becomes

$$
\begin{aligned}
\mathcal{L}_{s f}= & -\frac{3 \alpha}{4}\left(\xi+\frac{\bar{\gamma} \xi_{\eta}+\xi_{\gamma}-\xi}{\bar{\gamma}^{2}+1}\right)\left(\frac{h}{M_{P}}\right)^{2} \partial_{\mu} V^{\mu} \\
& -\frac{3}{4}\left(\beta \xi+\frac{(\bar{\gamma}+\beta)\left(\bar{\gamma} \xi_{\eta}+\xi_{\gamma}-\xi\right)}{\bar{\gamma}^{2}+1}\right)\left(\frac{h}{M_{P}}\right)^{2} \partial_{\mu} A^{\mu} .
\end{aligned}
$$

(iv) Equation (A7), in turn, must read

$$
\begin{aligned}
\frac{\Omega_{N}}{\Omega_{\mathrm{DM}}} \simeq & 3.2\left(\beta \xi+\frac{(\bar{\gamma}+\beta)\left(\bar{\gamma} \xi_{\eta}+\xi_{\gamma}-\xi\right)}{\bar{\gamma}^{2}+1}\right)^{2} \\
& \times\left(\frac{T_{\text {prod }}}{M_{P}}\right)^{3}\left(\frac{M_{N}}{T_{\text {prod }}}\right)^{2}\left(\frac{M_{N}}{10 \mathrm{keV}}\right),
\end{aligned}
$$

(v) In the discussion below Eq. (A7), the specific values of the nonminimal couplings $(1 / \bar{\gamma})=\xi_{\eta}=0$ should read $(1 / \bar{\gamma})=\xi_{\eta}=\beta=0$, and $\xi_{\gamma}=\xi, \xi_{\eta}=0$ should $\operatorname{read} \beta=\left[\left(-\xi_{\eta} \bar{\gamma}^{2}+\xi \bar{\gamma}-\xi_{\gamma} \bar{\gamma}\right) /\left(\xi \bar{\gamma}^{2}+\xi_{\eta} \bar{\gamma}+\xi_{\gamma}\right)\right]$.

The above changes do not affect the conclusions of Appendix A.

[1] M. Shaposhnikov, A. Shkerin, I. Timiryasov, and S. Zell, Einstein-Cartan gravity, matter, and scale-invariant generalization, J. High Energy Phys. 10 (2020) 177; Erratum, J. High Energy Phys. 08 (2021) 162.

[2] M. Shaposhnikov, A. Shkerin, I. Timiryasov, and S. Zell, Einstein-Cartan Portal to Dark Matter, Phys. Rev. Lett. 126, 161301 (2021).

Published by the American Physical Society under the terms of the Creative Commons Attribution 4.0 International license. Further distribution of this work must maintain attribution to the author(s) and the published articles title, journal citation, and DOI. 